21) Vgl. etwa den in BECK 1981 a, Anm. 9, zitierten Beleg.

22) Zur allgemeinen Wahrscheinlichkeit der "social career» einer sozialwissenschaftlichen Theorie vgl. GOULDNER 1974, Bd. 1, insb. S. 40f. - Ähnliches gilt wohl in bezug auf den Wechsel der Hintergrundannahmen von sozialwissenschaftlichen Theorien ("Paradigmenwechsel»).

23) Vgl. dazu etwa die Diskussion zwischen PARIS 1976 und OTTOMEYER 1976. - Ich sollte wohl wenigstens ein Beispiel einer empirischen Untersuchung angeben, welche in der Tendenz einem solchen theoretischen Ansatz entspricht: Man prüfe diesbezüglich HARVEY 1974, eine Untersuchung, in welcher der Autor mit Begriffen wie "Transmissionsagent", "objektive/subjektive Klassenlage» usw. arbeitet.

24) MAUKE 1971, S. 80.

\section{Literatur}

BECK, G., 1981 a: Darstellung und Kritik des verhaltens- und entscheidungstheoretischen Ansatzes in der Geographie. In: OSTHEIDER, M./STEINER, D. (Hg.): Theorie und Quantitative Methodik in der Geographie. Symposium, Zürich 1980. (Zürcher Geogr. Schriften; 1) Zürich, S. 119-139.

BECK, G. 1981 b: Der verhaltens- und entscheidungstheoretische Ansatz. Zur Kritik eines modernen Paradigmas in der Geographie. In: SEDLACEK, P. (Hg.): Kultur-/Sozialgeographie. Beiträge zu ihrer wissenschaftstheoretischen Grundlegung. (Uni-Taschenbücher; UTB 1053) Paderborn (im Druck).

BRUDER, K.-J., 1972: Verhalten als Funktion der Bedingungen von Verhalten. Zum Doppelcharakter der bürgerlichen Psychologie. In: BRINKMANN, H./BRUDER, K.-J./MÜNCH, R.: Wissenschaftstheorie + Gesellschaftliche Praxis. (edition 2000, theorie + kritik; 3) Gießen, S. 81-102.

BRUDER, K.-J., 1973: Entwurf der Kritik der bürgerlichen Psychologie. In: Ders. (Hg.): Kritik der bürgerlichen Psychologie. Zur Theorie des Individuums in der kapitalistischen Gesellschaft. (Bücher d. Wissens; Fischer-Tb. 6198) Frankfurt a. M., S. 92-217.

CORNFORTH, M., 1970: Marxistische Wissenschaft und antimarxistisches Dogma. (Marxist. Taschenbücher) Frankfurt a. M.

DEITERS, J./HEINRITZ, G. (Leitung), 1980: Fachsitzung «Aktionsräumliche Ansätze in der Zentralitätsforschung». In: SANDNER, G./NUHN, H. (Hg.): 42. Deutscher Geographentag Göttingen 1979. Tagungsbericht und wissenschaftliche Abhandlungen. (Verh.d. Dt. Geogr.-Tages; 42) Wiesbaden, S. 427-441.

EISEL, U., 1980: Die Entwicklung der Anthropogeographie von einer «Raumwissenschaft» zur Gesellschaftswissenschaft. (Urbs et Regio, Kasseler Schriften z. Geografie u. Planung; 17) Kassel.

GOULDNER, A. W., 1974: Die westliche Soziologie in der Krise. 2 Bde. (rowohlts dt. enzyklopädie; 360/361) Reinbek b. Hamburg. GRAUMANN, C. F., 1980: Verhalten und Handeln - Probleme einer Unterscheidung. In: SCHLUCHTER, W. (Hg.): Verhalten,
Handeln und System. Talcott Parsons' Beitrag zur Entwicklung der Sozialwissenschaften. (suhrkamp taschenbuch wissenschaft; 310) Frankfurt a. M., S. 16-31.

GREIFF, B.v., 1976: Gesellschaftsform und Erkenntnisform. Zum Zusammenhang von wissenschaftlicher Erfahrung und gesellschaftlicher Entwicklung. (Campus Diskussion: Krit. Sozialwiss.) Frankfurt/M., New York.

HABERMAS, J., 1970: Ein Literaturbericht (1967): Zur Logik der Sozialwissenschaften. In: Ders.: Zur Logik der Sozialwissenschaften. (ed. suhrkamp; 481) Frankfurt a. M., S. 71-310.

HARD, G., 1973: Die Geographie. Eine wissenschaftstheoretische Einführung. (Sammlung Göschen; 9001) Berlin, New York.

HARVEY, D., 1974: Klassenmonopolrente, Finanzkapital und Urbanisierung. In: Stadtbauwelt 41, S. 25-34. Wieder abgedruckt in MAYER, M./ROTH, R./BRANDES, V. (Hg.): Stadtkrise und soziale Bewegungen. Texte zur internationalen Entwicklung. (Polit. Ökonomie. Geschichte u. Kritik) Köln 1978, S. 55-77.

HÖLLHUBER, D., 1977: Modelle des wirtschaftenden Menschen in der Geographie. In: Wirtschaftsgeogr. Studien 1, S. 17-36.

HOLZKAMP, K., 1972: Kritische Psychologie. Vorbereitende Arbeiten. (Texte z. polit. Theorie u. Praxis; Fischer-Tb. 6505) Frankfurt a.M.

KASTER, T./LAMMERS, D. A., 1979: Ausgewählte Materialien zur Zeitgeographie. (Karlsruher Manuskripte z. Mathemat. u. Theoret. Wirtschafts- u. Sozialgeogr.; 35) Karlsruhe.

MÅRTENSSON, S., 1979: Der Einfluß regionaler Disparitäten auf die Zeitbudgets ausgewählter Haushaltstypen. In: KASTER/ LAMMERS 1979, S. 34-67.

MAUKE, M., 1971: Die Klassentheorie von Marx und Engels. (Krit. Studien z. Politikwiss.) 3.A. Frankfurt a. M.

MEINEFELD, W., 1977: Einstellung und soziales Handeln. (rororo studium; 103) Reinbek b. Hamburg.

OTTOMEYER, K., 1976: Antikritische Bemerkungen zu Rainer Paris. In: Gesellschaft (Hg.): Beiträge zur Marxschen Theorie 8/9. (ed. suhrkamp; 863) Frankfurt a. M., S. 335-349.

PARIS, R., 1976: Schwierigkeiten einer marxistischen Interaktionstheorie. Anmerkungen zu einem Programm der Vermittlung von Kritik der politischen Ökonomie und Interaktionstheorie. In Gesellschaft (Hg.): Beiträge zur Marxschen Theorie 7. (ed.suhrkamp; 827) Frankfurt a. M., S. 11-44.

THOMALE, E., 1974: Geographische Verhaltensforschung. In: DICKEL, H., u.a.: Studenten in Marburg. (Marburger Geogr. Schriften; 61) Marburg/Lahn, S. 9-30.

WIESSNER, R., 1978: Verhaltensorientierte Geographie. Die angelsächsische behavioral geography und ihre sozialgeographischen Ansätze. In: Geogr. Rundschau 30, 1978, S. 420-426.

WOLPERT, J., 1970: Eine räumliche Analyse des Entscheidungsverhaltens in der mittelschwedischen Landwirtschaft. In: BARTELS, D. (Hg.): Wirtschafts- und Sozialgeographie. (Neue Wiss. Bibliothek; 35) Köln, Berlin, S. 380-387.

\title{
Diskussion zum Vortrag Günther Beck
}

Diskussionsteilnehmer:

GB Dr. GÜNTHER BECK, Geograph, Göttingen (Referent)

KI Dr. KLAUS I. ITTEN, Geograph, Zürich (Diskussionsleiter)

PB PETER BƯNZLI, Geographiestudent, Zürich

PH Dr. PAUL HOYNINGEN, Wissenschaftsphilosoph, Zürich HW HERBERT WANNER, Geograph, Zürich

KI: Als Eröffnung der Diskussion möchte ich Ihnen folgende Frage stellen: In unserem Seminar zur Theorie in der Geographie haben wir in der letzten Sitzung über den Raumbegriff in der Geographie gesprochen. Welches ist nun Ihr Raumbegriff in der Verhaltensgeographie?
GB: Im behavioristischen Ansatz steht «Raum» gleichbedeutend für die Gesamtheit derjenigen Umweltgegebenheiten, die vom Individuum sinnlich wahrgenommen werden. Die Aufzeichnung solcher "Wahrnehmungsräume» ergibt jene in der Regel stark verzerrten Landschaftsbilder, wie sie in den bekannten "mental maps» der Perzeptionsforscher dargestellt sind. Im aktionistischen Ansatz bezeichnet «Raum» die Gesamtheit derjenigen Umweltgegebenheiten, die die "Bewegungs(spiel)räume» der Individuen bilden. Die Abbildungen solcher Räume sind von der Art der geometrischen Gebilde, wie sie 
z. B. die Time-Geographie in ihren Raum-ZeitModellen darstellt. Für den zuletzt angezeigten nicht-empiristischen Ansatz trägt es wohl nichts aus, vorgängig - d. h. vor der Aufstellung einer dem Objekt angemessenen Gesellschaftstheorie - einen Raumbegriff als fixe Kategorie zu definieren. Allerdings kann die Reflexion darüber auf der methodologischen Ebene ein Stück weit vorangetrieben werden. Das entsprechende Problem ließe sich etwa so formulieren, wie es HARVEY angegangen hat: nämlich das Räumliche als eine besondere Dimension des gesellschaftlichen Lebens begreifen zu müssen. Das meint, die Menschen sind in ihrem Verhalten eingebunden in ökonomische und soziale Situationen und Prozesse, welche sich durch eine je spezifische Räumlichkeit auszeichnen. So ist, um ein einfaches Beispiel zu geben, in einer privatwirtschaftlichen Gesellschaftsordnung das freie Feld hinterm Haus für den Bewohner möglicher «RegenerationsRaum», für den Finanzkapitalisten dagegen potentieller «Revenuen-Raum».

KI: Hat sich beispielsweise der Münchner Ansatz in der Verhaltensgeographie bewährt?

GB: Jüngere Selbsteinschätzungen der Münchner Schule zeigen, daß sie sich als Verhaltensgeographie versteht. Man kann diese Selbsteinschätzung kritisieren oder aufzeigen, daß die Münchner zumindest in ihren Anfängen weniger bewußt (moderne) Verhaltenswissenschaft betrieben als etwa andere Sozialgeographen. Gleichwohl, menschliches Verhalten hat die Münchner Sozialgeographie seit jeher angesprochen. Wir kennen ja alle das axiomatisierte Lehrgebäude, das auf den 7 «Grunddaseinsfunktionen» aufbaut: Wohnen, Arbeiten, Sich versorgen, ..., und in dem sich das Verständnis der Münchner von den Grundlagen räumlichen Verhaltens widerspiegelt. Indem die Münchner Sozialgeographen in ihren Arbeiten unter anderem die Beeinflußbarkeit menschlichen Verhaltens durch bestimmte Raumstrukturen aufgezeigt haben, hat sich ihre Schule einmal abgesehen von ihrer ideologischen Funktion - für die Planungspraxis «funktionsteiliger Gesellschaften» durchaus «bewährt». Wenn die Münchner Schule in Zukunft an praktischer Bedeutung einbüßen wird, so ist das primär nicht einer Unzulänglichkeit ihres wissenschaftlichen Lehrgebäudes zu verdanken, sondern vielmehr dem Umstand, daß die Erfordernis direkter Beeinflussung des Verhaltens großer Teile der Bevölkerung (und nicht der mittelbaren über den «Umweg» räumlicher Determinanten) in der «Funktionsgesellschaft» wächst und somit andere verhaltenswissenschaftliche Ansätze favorisiert.

PH: Ich habe eine Frage im Zusammenhang mit dem, was Sie über Gesetze gesagt haben. Zunächst die beiden Beispiele, die Sie genannt haben: «Menschen verhalten sich gemäß Anspruchsniveau und
Nutzenmaximierung». Dem haben Sie noch den Ehrentitel von «nomologischen Hypothesen» gegeben. Ich würde dort mit der Kritik noch schärfer ansetzen und sagen, das sind doch wahrscheinlich analytische Sätze, die überhaupt keinen Erfahrungsgehalt haben. Nutzenmaximierung des Verhaltens, natürlich, wenn ich im Nachhinein sage, was Nutzen ist, wird es immer wahr, ebenso, wenn ich über Anspruchsniveau rede. Wenn ich frage, was heißt Anspruchsniveau überhaupt, dann werde ich sagen müssen, ja das, was sich im Verhalten zeigt. Also diese Sätze sind genau so solide wie Sätze: «Alle Junggesellen sind unverheiratet». Das liegt im Begriff des Junggesellen, das liegt im Begriff des Anspruchsniveaus, das liegt im Begriff dieser Nutzenvorstellung, daß man diese Sätze formulieren kann. Das sind keine nomologischen Hypothesen, sondern allenfalls Begriffsexplikationen. Also ich würde da mit der Kritik viel weiter gehen und sagen, daß solche Sätze den Gesetzesstatus gar nicht erreichen können.

Nun möchte ich noch etwas sagen, wo mir etwas merkwürdig vorgekommen ist: Sie sagten, daß man in der Naturwissenschaft Gesetze deswegen haben muß, um Voraussehbarkeit und Beherrschung der Natur zu bekommen; wenn man dasselbe in der Verhaltenswissenschaft verwendet, entsprechend Voraussehbarkeit und Beherrschung des Menschen. Habe ich Sie da richtig verstanden?

GB: Ja, die Kritik ging vom Interesse der modernen Naturwissenschaften aus...

PH: Und geht das Argument jetzt wirklich so: Wenn ich abstrahierend Gesetze der Natur formuliere, die Voraussehbarkeit und Beherrschung ermöglichen, und wenn ich dasselbe mache in den Verhaltenswissenschaften, dann bekomme ich Voraussehbarkeit und die Beherrschung des Menschen?

GB: Umgekehrt, das Interesse an Voraussehbarkeit und Beherrschung bewirkt das Interesse an diesen Gesetzen.

PH: Das möchte ich dann sehr bezweifeln und zwar deshalb, weil nomologische Hypothesen beim Menschen nicht nur zur Beherrschung und Knebelung, sondern durchaus auch für emanzipatorische Prozesse benutzt werden können. Bei der Natur geht es deshalb nicht, weil diese ihre Gesetze nicht zur Kenntnis nehmen kann. Ich gebe Ihnen ein Beispiel. «In spätkapitalistischen Gesellschaften haben Kinder aus der Unterschicht durch die Ausbildung wesentlich schlechtere Sprachchancen.» Wenn ich eine solche nomologische Hypothese hinschreibe und verifiziere, dann kann das natürlich gerade Instrument sein, um diese Chancenungleichheit abzuschaffen. Oder wenn Sie das individuelle Verhalten nehmen, z. B. wenn Sie einem Zwangsneurotiker klarmachen, was ihm hilft, sich von seinen Zwängen zu befreien, indem er sich von bestimmten Situatio- 
nen fern hält. Also: es scheint mir nur ein rhetorischer Trick zu sein und kein Argument, wenn man sagt, daß die nomologischen Hypothesen in der Verhaltenswissenschaft zur Beherrschung des Menschen gebraucht werden. Dann kann man noch zwei Stammväter unterschiedlicher Provenienz anführen: der eine ist POPPER, der andere ist HEGEL.

GB: Können Sie mir eine nomologische Hypothese der Verhaltenswissenschaftler angeben, bei der so formuliert wurde? Eben haben Sie nämlich meines Erachtens keine angegeben. Das Beispiel mit den Kindern aus der Unterschicht ist ja keine nomologische Hypothese (sondern eine durchaus lohnende sog. Quasi-Hypothese), weil darin eine bestimmte Gesellschaft genannt ist. Sie geben ja keine Hypothese an, daß sich Menschen eines bestimmten Alters und einer bestimmten Schicht aufgrund der Ausbildung immer und überall so "verhalten». Das heißt, Sie müßten in Ihrer Hypothese (oder in einer übergeordneten) irgendwo auf die Natur des Menschen zurückkommen, aus der dann die Verhaltensweise dieser Kinder abzuleiten wäre ${ }^{1)}$. Dagegen haben Sie von vornherein den Konnex zu einer bestimmten historischen Situation gezogen. Sie haben gesagt, «Kinder in der spätkapitalistischen Gesellschaft», und die Frage aufgeworfen, durch welche Faktoren die Variation des Verhaltens von Kindern im Spätkapitalismus bedingt ist. Das ist genau der Punkt, wo ich mit einer nicht-verhaltenswissenschaftlichen Gesellschaftstheorie ansetze. Mit Ihrer Aussage aber ist gar keine nomologische Hypothese formuliert.

PH: Ich glaube schon, wenn man den Satz auffaßt als: "In allen spätkapitalistischen Gesellschaften...». Aber das ist vielleicht nicht so wichtig.

GB: Aber Ihre Frage ging noch ein bißchen woanders hin. Die Frage war, was führt denn den Verhaltenswissenschaftler dazu, nach solchen Gesetzen zu suchen. Das kann sicherlich mal ein emanzipatorisches Interesse sein, ist es aber im allgemeinen nachweislich in dem Sinne nicht, daß bei allen diesen Versuchen, solche nomologischen Hypothesen zu gewinnen, immer von den entsprechenden gesellschaftlichen Verhältnissen, wie Sie sie selbst aufgeführt haben, abstrahiert wird. Dieses Verfahren führt dazu, daß dann hinterher solche überhistorischen Aussagen über die Mensch-Natur, wie ich sie in meinem Referat aufgeführt habe, herauskommen. Und diese Mensch-Natur gilt - voraussetzungsgemäß - als (in historischen Zeiträumen) nichtveränderlich; die gesellschaftlichen Verhältnisse, durch die ein bestimmtes Verhalten zum Tragen kommt, aber wären veränderbar.

Andererseits verzichtet eine bestimmte Gruppe von Geographen meist auch noch darauf, solche Gesetze $\mathrm{zu}$ formulieren. Sie machen es so, daß sie einfach Regelmäßigkeiten im Verhalten von Gruppen von Menschen aufzeigen und sagen: «Diese bestimmte
Gruppe von Käufern verhält sich so (besucht zum Beispiel häufig diesen Supermarkt)». Dies beschreibt eine gewisse Invarianz, richtig. Die Frage ist nur, weshalb wird beschrieben, daß Sie und ich zu den $32 \%$ gehören, die bei diesem Supermarkt ankommen, weil wir ein Auto besitzen, und andere nicht dort ankommen, weil sie kein Auto besitzen. Das sind die Verfahren, nein das offenbart die Interessen, da liegt nichts mehr im Emanzipatorischen. Für Sie ist es auch keine Erkenntnis, zu wissen, daß Sie zu den $32 \%$ gehören, die diesen Supermarkt besuchen. Das wissen Sie von vornherein. Ihr Verhalten, wenn es sich gar noch zu einer «Gruppe gleichen Verhaltens» aggregieren läßt, bezeichnet man als «invariantes Verhalten» vor allem dann, wenn Sie es längere Zeit durchhalten. $\mathrm{Da}$ gibt es ein Interesse, vorauszusagen, daß diese oder jene Gruppe sich auch weiterhin so verhalten wird. Wenn ein paar Menschen aus dieser Gruppe ausscheiden oder andere hinzukommen oder die Gruppe unverändert bleibt, wen interessiert das? Bringt das für die Menschen einen Erkenntnisgewinn? Oder gar einen emanzipatorischen Gewinn? Den müßte man schon konstruieren. Über den Weg solcher Gruppenbildungen, über das Aufzeigen solchen massenhaften Verhaltens, steuert man im übrigen oft diese nomologischen Hypothesen an. Aber ich kenne keine, wie wir sie gerne hätten.

PH: Dann machen die Leute einfach schlechte Wissenschaft. Der Ansatz ist damit noch nicht diskreditiert.

GB: Mich interessiert, was sie dabei eliminieren, mit welchen Methoden sie dabei vorgehen. Aber selbst wenn sie mit anderen Methoden diese Verhaltensforschung «besser» betreiben würden, würde der Charakter meiner Kritik bestehen bleiben müssen. Dies ist, wie mir scheint, auch in der Wissenschaftstheorie eine stehende Kontroverse. Mir geht es aber nicht darum, allgemeine Wissenschaftstheorie zu betreiben, sondern konkrete Wissenschaftskritik. Es ist für die Situation unserer Gesellschaft von Bedeutung, was die Wissenschaftler, die sich Geographen nennen, für Arbeiten machen. Deswegen versuche ich, konkrete Wissenschaftskritik zu machen.

HW: Im Theorieseminar haben wir ausgehend vom Kapitel über die Umweltwahrnehmung im Buch von HARD die Frage diskutiert, wie man an eine Forschungsfront gelangen kann. Können Sie uns etwas darüber berichten, wie Sie in der Verhaltensgeographie diesbezüglich vorgegangen sind?

GB: Damit setzen Sie selber voraus, daß die Verhaltensgeographie eine Forschungsfront darstellt. Nehmen wir ferner an, daß ich mich darin eingereiht hätte, bleibt die Frage, wie? Nun, sicher dadurch, daß ich zur Kenntnis nehmen mußte, was da in der Geographie seit einigen Jahren vor sich geht. Es ist ja 
nicht so, daß es seit jeher eine Verhaltensgeographie gibt. Erst als im Zuge der sog. quantitativen und theoretischen Revolution in der Geographie mit der Kategorie des Menschen als "handelndes Wesen» (entsprechend MAX WEBERs Konzeption) ein neues Menschenbild in das Fach übernommen wurde, ist die Geographie (moderne) "Verhaltenswissenschaft» geworden. Jeder, der heute mit Geographie beschäftigt ist, wird zur Kenntnis nehmen müssen, daß er mit dieser Verhaltensgeographie (im weitesten Sinn verstanden) zu tun hat, es sei denn, er vertritt einen abgelebten Forschungsansatz und kümmert sich nicht um das, was um ihn herum vor sich geht. Damit ist aber auch eine Affinität zu den Erkenntnisproblemen und Methodenfragen der allgemeinen Wissenschaftstheorie gegeben. Und damit sind Sie mitten drin in der Diskussion, auch in derjenigen, die jenseits der Grenzen der Geographie geführt wird. Sie können sich natürlich als einzelner dieser Situation willentlich entziehen, indem Sie etwa vorgeben, «bloß empirisch» zu arbeiten, oder indem $\mathrm{Sie}$ - wie gesagt - traditionelles Gedankengut pflegen. Die Geographie insgesamt hat da wohl keine Alternative.

HW: Mich interessiert auch noch, wo und wie Sie sich orientiert haben. Also in welcher Literatur Sie diese Verhaltensansätze studiert haben.

GB: Was man verfolgen muß, ist neben der geographischen Literatur die Diskussion in der Soziologie und vor allem in der Psychologie sowie diejenige in der allgemeinen Wissenschaftstheorie. Wichtig scheint mir allerdings dabei, daß man Position beziehen muß, wenn man widerstreitende Ansätze erkennen will, und weil ansonsten die politische Dimension dieser Diskussion nicht erkannt und sie als bloß «theoretische» Auseinandersetzung verstanden würde.

KI: Von welcher Schule sind Sie besonders beeinflußt worden? Ist es beispielsweise jene von TORSTEN HÄGERSTRAND oder jene von WALDO TOBLER?

GB: Außer der Hägerstrand-Schule gibt es in der jüngeren Geographie ja kaum das, was man als «Schule» im herkömmlichen Sinn versteht. Die Hägerstrand-Schule hat mit der Time-Geography allerdings eine neue Konzeption entwickelt - ich hänge ihr nicht an. Entscheidender für die Prägung der eigenen wissenschaftlichen Position sind wohl im allgemeinen jene losen Diskussionszusammenhänge, in denen der einzelnen von uns jeweils steht.

PH: Sie sagten, daß Theorien aufgrund des Einbezugs von Störfaktoren nicht falsifizierbar seien und deshalb das Auswechseln von Theorien wissenschaftsexterne Gründe habe. Beziehen Sie das nur auf die Geographie oder auf alle Wissenschaften? Falls Sie es auf beide Fälle beziehen, haben Sie ge- wisse Gewährsleute, auf welche Sie sich beziehen? THOMAS KUHN kann es nicht sein.

GB: Es ist richtig, daß ich damit einen Hinweis geben wollte auf das praktische Interesse, welchem der Wissenschaftler immer irgendwie ausgesetzt ist. Es gibt ja in der Wissenschaft auch eine berufliche Karriere, zu der eine "neue» Geographie vielen verhilft. Dies wäre ein Beispiel für einen wissenschaftsexternen Grund zum Auswechseln der Theorie. Daß natürlich auch eine ganze Reihe von wissenschaftsinternen Gründen diskutiert wird, ist mir bekannt, aber in meinem Vortrag vielleicht zu kurz gekommen. Es wäre in diesem Rahmen etwas abseitig gewesen, auf diese Diskussion einzugehen. Dennoch bin ich an einer Stelle meiner Ausführungen auf diese Diskussion eingegangen. Ich habe ja zwei verschiedene Theorien miteinander verglichen: die eine nimmt die (Verhaltens-)Bedingung der Klassenlage eines Menschen in ihre Hypothesen auf, die andere abstrahiert davon. Im Sinne von LAKATOS stehen sich zwei verschiedene Theorien gegenüber, werden miteinander verglichen. Vorzuziehen wäre, dem Wissenschaftsfortschritt zuliebe, diejenige Theorie, welche einen zusätzlichen Erkenntnisgewinn garantierte. Da ist die (normative) Logik von LAKATos, aber noch lange kein Grund, der in der Praxis des Wissenschaftsbetriebes entscheidend ist. Für entscheidend halte ich vielmehr, welche Funktion eine Theorie in bezug auf eine bestimmte Gesellschaft hat. Dies meinte ich, wenn ich von «externen Gründen» sprach.

In diesem Zusammenhang interessiert mich vor allem die aktuelle Entwicklung in der Geographie. Weshalb gibt es in der Geographie nicht nur einen Paradigmenwechsel im Sinne des Wechsels der (formalen) Methoden, sondern auch einen Wechsel bei den inhaltlichen Ansätzen? Mit der Behavioral Geography, der Welfare Geography, der Humanistic Geography, der Radical Geography und anderen «Geographies» ist ein ganzer Strauß von Ansätzen im Fach aufgetaucht. Diese Entwicklung wissenschaftsintern zu begründen, fällt mir schwer. Und das Aufkommen dieses Straußes etwa speziell mit dem Genie von Geographen zu begründen, fällt mir auch schwer und würde darüber hinaus überhaupt nicht erklären können, weshalb diese Ansätze zu einem bestimmten Zeitpunkt in der Geographie aufgekommen sind. Ich würde da eher eine wissenschaftsexterne Begründung geben. Der Vietnamkrieg der USA war sicherlich einer der Gründe, er gab den Anstoß für viele Wissenschaftler, nach «neuen Theorien» Ausschau zu halten oder solche zu konstruieren. Die genannten Ansätze, welche alle aus Amerika zu uns kommen, sind mit einer bestimmten Funktion verbunden, die es im einzelnen zu prüfen gälte. Als Strau $\beta$ aber könnten sie einen ziemlich unerfreulichen Effekt haben, indem sie nämlich fälsch- 
licherweise den Eindruck erwecken, daß durch die Vielfalt der Ansätze die Bedingung für eine zunehmend objektivere Erkenntnis in der Geographie geschaffen sei. - Dies verstehe ich als einen ganz groben Interpretationsversuch.

PB: Entspricht dieser Strauß von Ansätzen einer «Normalwissenschaft»? Oder müßte man nicht vielmehr von einer "permanenten Revolution» sprechen?

GB: Als letzte Normalphase in der deutschsprachigen Geographie müßte man wohl die Zeit ansehen, in der das Landschaftskonzept dominierte. Innerhalb dieser Landschaftsgeographie fanden keine Problemverschiebungen mehr statt. Die Landschaftsgeographen haben eigentlich gar kein Problem mehr entdeckt. Sie haben im Grunde genommen immer am gleichen Problem gearbeitet, nur empirisch haben sie es immer wieder anders gemacht. Und mit einem Mal war auch ihre ideologische Funktion politisch nicht mehr gefragt. Im Zuge der quantitativ-theoretischen Revolution entstand jene Vielzahl von neuen Ansätzen, von denen ich schon sprach, vermutlich auch deshalb, weil eben die Probleme plötzlich «vielzählig» waren. Nur: dies geschah nicht in der deutschsprachigen Geographie, sondern vor allem in Amerika, und da geht die «Revolution» in der Tat vorerst weiter.

KI: Könnten Sie uns noch etwas erzählen über die Verhältnisse der Geographie in der BRD?

GB: Wenn ich es richtig sehe, gibt es in der deutschen Geographie praktisch keine Forschergruppe, welche originär (Verhaltens-)Theorien entwickelt. Die deutschen Geographen profitieren von der amerikanischen Behavioral Geography und von mehr aktionstheoretisch arbeitenden Gruppen wie derjenigen um HÄGERSTRAND.

KI: Aber ich glaube, man darf sich da kein falsches Bild machen. Was Sie darstellen, ist eine Forschungsfront, die auf einige wenige Exponenten beschränkt ist. Aber in der BRD ist doch die Länderkunde wieder stark im Kommen.

GB: Die Frage ist, wieso in der BRD die Länderkunde wieder gepflegt wird. Das hat sicher gesellschaftspolitische Gründe. Es hat auch Gründe, die in der Tradition des Faches liegen, d. h. in der Struk- tur jener Kommune, die auf der Universität Geographie lehrt. Die Mehrzahl der Vertreter der Länderkunde hat ein Jahrzehnt geschwiegen zu den Angriffen der kritischen Quantitativen und Theoretischen Geographie. Auch die Schüler der traditionellen Länderkundler haben sich nicht zu Wort gemeldet. Jetzt besteht wieder die Möglichkeit, sich im politischen Aufwind zu Wort zu melden, über die Ministerien, über die Verordnungen von Schullehrplänen, über Eingriffe in die Studienlehrpläne usw. Doch sie gehen einer Diskussion weitgehend aus dem Wege. Sie machen nicht das, was ich vorhin als erforderlich hinstellte: sie nehmen nicht den länderkundlichen Standpunkt ein und setzen sich mit der Entwicklung in der Geographie auseinander. Sie nehmen den länderkundlichen Standpunkt ein, und dabei bleibt's. Bleibt die Frage, was macht diesen Standpunkt plötzlich wieder hoffähig?

Dazu kommt die Struktur der Geographischen Institute. Untersuchen Sie diese Struktur, dann werden Sie sehen, daß in der Altersverteilung ziemliche Brüche bestehen. Da gibt es die stark vertretene Generation der um 50jährigen. Die meisten von ihnen sind Schüler der traditionellen Länderkundler. Sie haben nicht in der quantitativ-theoretischen Revolution drin gestanden. Das wären die heute um 40jährigen. Diese sind auf den Lehrstühlen in geringerer Zahl vertreten. Die institutionellen Strukturen sind also relativ festgelegt, die Denkstrukturen auch. Das klingt pejorativ, soll es aber nicht sein. Denn «an sich» brauchte man von einem 40jährigen nicht zu verlangen, daß er immer hinter den neuesten Theorien herjagen muß. Es könnte ja, wiederum «an sich», für die Entwicklung von Wissenschaft sinnvoll sein, wenn ich als 40jähriger dabei bliebe, was ich gelernt habe. Mit 40, so sagen denn manche, sollte man seinen Standpunkt gewonnen haben. Das sei dann effektiv auch für die Gesellschaft.

KI: Das entläßt uns, glaube ich, recht hoffnungsvoll, weil der Prozeß die Probleme automatisch löst.

1) Anmerkung: Der letzte Satz enthält eine nicht-notwendige Ergänzung zu dem zuvor Gesagten und ist deshalb überflüssig bzw. hier unzutreffend; er resultiert aus der von mir nicht genügend beachteten Trennung von Universalität (dem Grad nach abnehmend von: alle Menschen - Kinder - Unterschichtenkinder) und Generalität (dem Grad nach abnehmend von: immer und überall - im Spätkapitalismus - im Wien der 30er Jahre) einer Aussage. 